\title{
Fractional topological superconductivity and parafermion corner states
}

\author{
Katharina Laubscher ${ }^{\circ}$, Daniel Loss $\odot$, and Jelena Klinovaja \\ Department of Physics, University of Basel, Klingelbergstrasse 82, CH-4056 Basel, Switzerland
}

(Received 5 May 2019; published 11 November 2019)

\begin{abstract}
We consider a system of weakly coupled Rashba nanowires in the strong spin-orbit interaction (SOI) regime. The nanowires are arranged into two tunnel-coupled layers proximitized by a top and bottom superconductor such that the superconducting phase difference between them is $\pi$. We show that in such a system strong electron-electron interactions can stabilize a helical topological superconducting phase hosting Kramers partners of $\mathbb{Z}_{2 m}$ parafermion edge modes, where $m$ is an odd integer determined by the position of the chemical potential. Furthermore, upon turning on a weak in-plane magnetic field, the system is driven into a second-order topological superconducting phase hosting zero-energy $\mathbb{Z}_{2 m}$ parafermion bound states localized at two opposite corners of a rectangular sample. As a special case, zero-energy Majorana corner states emerge in the noninteracting limit $m=1$, where the chemical potential is tuned to the SOI energy of the single nanowires.
\end{abstract}

DOI: 10.1103/PhysRevResearch.1.032017

Introduction. The search for topological phases of matter has generated an enormous amount of research. Motivated by the discovery and classification of topological insulators (TIs) and topological superconductors (TSCs), the field has been driven by the desire to access phases with increasingly exotic properties. In particular, it has been found that the effects of strong electron-electron interactions can lead to exotic fractionalized phases, which are considered particularly interesting due to their potential use for topological quantum computation. However, only one-dimensional (1D) systems allow for an analytically tractable description of such strong interactions via the bosonization formalism. In order to study strongly interacting systems in more than one dimension, one therefore resorts to the so-called coupled-wire approach, where two- or three-dimensional systems are built up from weakly coupled 1D channels, such as nanowires. This approach has proven to be exceptionally fruitful in accessing the fractional counterparts of several well-known topological phases such as fractional quantum Hall states [1-3], fractional TIs and TSCs [4-15], as well as fractional spin liquids [16-18].

Recently, a lot of interest has been raised by the generalization of conventional TIs/TSCs to so-called higherorder TIs/TSCs [19-39]. While a conventional $d$-dimensional TI/TSC exhibits $(d-1)$-dimensional gapless boundary modes, a $d$-dimensional $n$ th-order TI/TSC hosts gapless modes at its $(d-n)$-dimensional boundaries. While electronelectron interactions have been taken into account in a few cases $[40,41]$, the main focus was on noninteracting systems, neglecting the possible existence of exotic fractional phases supporting emergent parafermions. This raises the question

Published by the American Physical Society under the terms of the Creative Commons Attribution 4.0 International license. Further distribution of this work must maintain attribution to the author(s) and the published article's title, journal citation, and DOI. whether a coupled-wire approach can be used to extend the class of higher-order topological phases to the fractional regime. In this Rapid Communication, we show that this is indeed possible and explicitly construct a two-dimensional (2D) fractional second-order TSC exhibiting exotic parafermion corner states.

Our model consists of two layers of coupled Rashba nanowires with proximity-induced superconductivity of a phase difference of $\pi$ between the upper and lower layers (see Fig. 1). In a first step, we show that in the presence of strong electron-electron interactions, such a setup exhibits a helical topological superconducting phase with gapless helical $\mathbb{Z}_{2 m}$ parafermion edge modes propagating along the edges. Here, $m$ is an odd integer determined by the position of the chemical potential $\mu$. In the special case $m=1$, where $\mu$ is tuned to the spin-orbit interaction (SOI) energy of the single nanowires, Majorana edge modes emerge even in the noninteracting regime. At lower densities, the fractional regime $m>1$ emerges in the presence of strong electronelectron interactions as the SOI and Fermi wave vectors get commensurable.

In a second step, we include a small time-reversal breaking perturbation in the form of a weak in-plane magnetic field to gap out the helical edge modes. For a finite rectangular sample, we find $\mathbb{Z}_{2 m}$ parafermions localized at two opposite corners of the system depending on the direction of the magnetic field, which places our model in the class of 2D fractional second-order TSCs. Unlike most examples of higher-order topological phases, the stability of these corner states does not rely on spatial symmetries but is guaranteed by particle-hole symmetry alone. Also, the parafermion corner states found here emerge in a spatially uniform 2D system, while in previous studies parafermions have been constructed as bound states localized at the interfaces of nonuniform 2D systems [42-49] or at the ends of 1D wires [50-57].

Model. We consider two layers of coupled Rashba nanowires proximitized by bulk $s$-wave superconductors (see Fig. 1). Each nanowire of length $L$ is modeled by a 


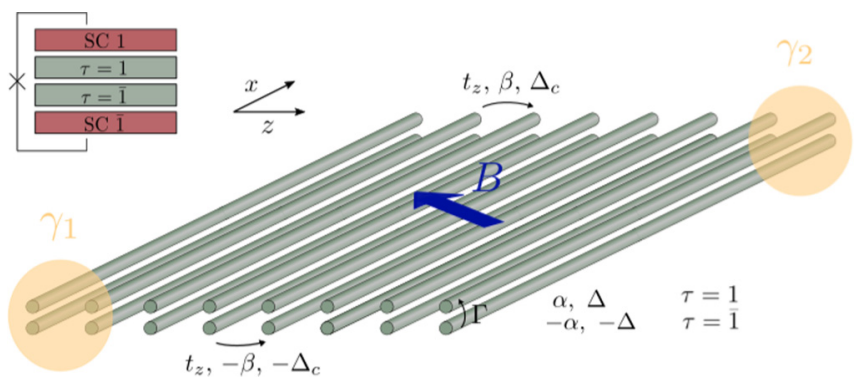

FIG. 1. The setup consists of two layers of coupled Rashba nanowires where the index $\tau=1(\tau=\overline{1})$ denotes the upper (lower) layer. The strength of the Rashba SOI associated with propagation along the $x$ direction is given by $\alpha(-\alpha)$ for the upper (lower) layer. Both layers are brought into proximity to an $s$-wave bulk superconductor such that there is a phase difference of $\pi$ between them. In addition, the two layers are strongly coupled by interlayer tunneling of strength $\Gamma$. Neighboring nanowires of the same layer are weakly coupled via a spin-conserving hopping term of strength $t_{z}$, via a spin-flip hopping term of strength $\beta(-\beta)$ associated with Rashba SOI along the $z$ direction, and via a crossed-Andreev superconducting term of strength $\Delta_{c}\left(-\Delta_{c}\right)$, where the last two terms are again of opposite sign for the two layers. Finally, a weak in-plane magnetic field of strength $B$ is applied. We show that in specific regions of parameter space, this system hosts two zero-energy corner states (here denoted by $\gamma_{1}$ and $\gamma_{2}$ ) at two opposite corners of a rectangular sample. In the noninteracting case, these states are Majorana zero modes, whereas strong electron-electron interactions lead to $\mathbb{Z}_{2 m}$ parafermion corner states, where $m$ is an odd integer depending on the position of the chemical potential.

free-particle Hamiltonian

$$
H_{0, n}=\sum_{\tau, \sigma} \int d x \psi_{n \tau \sigma}^{\dagger}\left[-\frac{\hbar^{2} \partial_{x}^{2}}{2 m}-\mu+i \alpha \tau \sigma \partial_{x}\right] \psi_{n \tau \sigma}
$$

Here, $\psi_{n \tau \sigma}^{\dagger}(x)\left[\psi_{n \tau \sigma}(x)\right]$ creates (destroys) an electron at position $x$ in the $n$th wire in the layer $\tau \in\{1, \overline{1}\}$ of spin $\sigma \in\{1, \overline{1}\}$, where we define the spin quantization axis along the SOI direction. The Rashba coefficient $\alpha$ is taken to be of equal magnitude for all nanowires, but of opposite sign for the two layers. The SOI energy associated with propagation along the nanowire is $E_{s o}=\hbar^{2} k_{s o}^{2} /(2 m)$ for $k_{s o}=m \alpha / \hbar^{2}$, and the chemical potential $\mu$ is defined relative to $E_{s o}$. The proximityinduced superconductivity is described by

$$
H_{\Delta, n}=\Delta \sum_{\tau} \tau \int d x \psi_{n \tau 1} \psi_{n \tau \overline{1}}+\text { H.c. }
$$

where we have set the phase difference between the two superconductors to $\pi$. This can, for example, be realized by the Josephson-junction setup shown in the inset of Fig. 1, where the phase difference between the two superconductors is adjusted by controlling the magnetic flux through the superconducting loop [58,59]. Alternatively, a thin insulating layer of randomly oriented magnetic impurities [60] could be placed between one of the layers and the corresponding superconductor such that the phase difference of $\pi$ arises due to spin-flip tunneling via the impurities [61-64]. Furthermore, the two layers are coupled by interlayer tunneling of the form

$$
H_{\Gamma, n}=\Gamma \sum_{\tau, \sigma} \int d x \psi_{n \tau \sigma}^{\dagger} \psi_{n \bar{\tau} \sigma},
$$

such that the total Hamiltonian describing an effective double nanowire (DNW) composed of two strongly coupled nanowires from different layers is given by $H_{n}=H_{0, n}+$ $H_{\Delta, n}+H_{\Gamma, n}$. Finally, the DNWs are weakly coupled via a spin-conserving hopping term of strength $t_{z}$, via a spin-flip hopping term of strength $\beta(-\beta)$ associated with Rashba SOI along the $z$ direction, as well as via a crossed-Andreev superconducting term of strength $\Delta_{c}\left(-\Delta_{c}\right)$, where the last two terms are again of opposite sign for the two layers. Here, $\left|t_{z}\right|,|\beta|,\left|\Delta_{c}\right| \ll|\Delta|,|\Gamma|$. The interwire Hamiltonian can then be written as

$$
\begin{aligned}
H_{\perp}= & \sum_{n, \tau, \sigma, \sigma^{\prime}} \int d x\left\{\Delta_{c} \tau \psi_{n \tau \sigma}\left(i \sigma_{y}\right)_{\sigma \sigma^{\prime}} \psi_{(n+1) \tau \sigma^{\prime}} / 2\right. \\
& \left.+\psi_{n \tau \sigma}^{\dagger}\left[-t_{z} \delta_{\sigma \sigma^{\prime}}-i \beta \tau\left(\sigma_{x}\right)_{\sigma \sigma^{\prime}} / 2\right] \psi_{(n+1) \tau \sigma^{\prime}}\right\} \\
& + \text { H.c. }
\end{aligned}
$$

The total Hamiltonian is now given by $H=$ $\sum_{n} H_{n}+H_{\perp}$, which in momentum space takes the form $H=\frac{1}{2} \sum_{k_{z}} \int d k_{x} \Psi_{\mathbf{k}}^{\dagger} \mathcal{H}(\mathbf{k}) \Psi_{\mathbf{k}}$ in the basis $\Psi_{\mathbf{k}}=$ $\left(\psi_{\mathbf{k} 1 \uparrow}, \psi_{\mathbf{k} 1 \downarrow}, \psi_{-\mathbf{k} 1 \uparrow}^{\dagger}, \psi_{-\mathbf{k} 1 \downarrow}^{\dagger}, \psi_{\mathbf{k} \hat{1} \uparrow}, \psi_{\mathbf{k} \overline{1} \downarrow}, \psi_{-\mathbf{k} \overline{1} \uparrow}^{\dagger}, \psi_{-\mathbf{k} \overline{1} \downarrow}^{\dagger}\right)$ with

$$
\begin{aligned}
\mathcal{H}(\mathbf{k})= & {\left[\frac{\hbar^{2} k_{x}^{2}}{2 m}-2 t_{z} \cos \left(k_{z} a_{z}\right)-\mu\right] \eta_{z}-\alpha k_{x} \tau_{z} \sigma_{z} } \\
& +\beta \sin \left(k_{z} a_{z}\right) \tau_{z} \sigma_{x}+\Gamma \tau_{x} \eta_{z} \\
& +\left[\Delta+\Delta_{c} \cos \left(k_{z} a_{z}\right)\right] \tau_{z} \eta_{y} \sigma_{y} .
\end{aligned}
$$

Here, $\tau_{i}, \eta_{i}$, and $\sigma_{i}$ for $i \in\{x, y, z\}$ are Pauli matrices acting in layer, particle-hole, and spin space, respectively, and $a_{z}$ is the spacing between neighboring nanowires. The system belongs to the symmetry class DIII [65] with time-reversal (particlehole) symmetry given by $\mathcal{T}=i \sigma_{y} \mathcal{K}\left(\mathcal{P}=\eta_{x} \mathcal{K}\right)$.

Helical topological superconducting phase. We now demonstrate that the system can be brought into a helical topological superconducting phase hosting two counterpropagating $\mathbb{Z}_{2 m}$ parafermion edge modes in the presence of strong electron-electron interactions. For this, we follow the method developed before for fractional TIs [4,8]: First, we solve the DNW Hamiltonian $H_{n}$ and demonstrate that, due to the interplay between $\Delta$ and $\Gamma$, the elementary excitations are given by gapless $\mathbb{Z}_{2 m}$ parafermion modes. We note that, in contrast to Refs. $[4,8]$, there are two competing gap-opening mechanisms, such that when the system is brought close to the critical point $\Gamma \approx \Delta$, again half of the modes are left gapless. Second, we include weak hoppings between DNWs to gap out the parafermion modes in the bulk but leave Kramers pairs of gapless parafermion modes at the edges of the system. Again, if $\beta$ and $\Delta_{c}$ counterbalance each other, the edge modes propagating along the $x$ axis are perfectly localized at the outermost DNWs. Importantly, the topological phase is robust against deviations from these fine-tuned points, which will, however, lead to increased localization lengths of the edge states. 
For illustrative purposes, we first consider the noninteracting regime with $m=1$ and set $\mu=0$. To treat the DNW Hamiltonian $H_{n}$, we linearize the spectra of the single nanowires around the Fermi points [66] as $\psi_{n \tau \sigma}(x)=$ $R_{n \tau \sigma}(x) e^{i k_{F}^{1 \tau \sigma} x}+L_{n \tau \sigma}(x) e^{i k_{F}^{\mathrm{I} \sigma} x}$, where $R_{n \tau \sigma}(x), L_{n \tau \sigma}(x)$ vary slowly on the scale of $k_{s o}^{-1}$ and the Fermi momenta are given by $k_{F}^{r \tau \sigma}=(\sigma \tau+r) k_{s o}$ [67]. We note that upon a change of basis defined by $\bar{L}_{n \kappa \nu}=\left(L_{n \kappa \nu}-i \kappa \nu L_{n \bar{\kappa} \overline{\bar{\nu}}}\right) / \sqrt{2}, \bar{R}_{n \kappa \nu}=$ $\left(R_{n \kappa \nu}-i \kappa \nu R_{n \bar{\kappa} \bar{\nu}}\right) / \sqrt{2}, H_{n}$ takes a block-diagonal form, while the structure of the Fermi momenta remains unchanged. For $\Delta \neq 0$, the exterior branches $\bar{R}_{n \kappa \kappa}$ and $\bar{L}_{n \kappa \bar{\kappa}}$ are fully gapped by superconductivity, whereas the interior branches $\bar{L}_{n \kappa \kappa}$ and $\bar{R}_{n \kappa \bar{\kappa}}$ have two competing gap-opening mechanisms given by interlayer tunneling and superconductivity. In the following, we thus focus on the interior branches only and tune the system to the critical point $\Delta=\Gamma$. In the new basis, the superconducting and tunneling terms take the forms $H_{\Gamma, n}=i \Gamma \sum_{\kappa} \int d x \bar{R}_{n \bar{\prime}}^{\dagger} \overline{\bar{L}}_{n \kappa \kappa}+$ H.c., $H_{\Delta, n}=$ $\Delta \sum_{\kappa} \int d x \bar{R}_{n \kappa \bar{\kappa}}^{\dagger} \bar{L}_{n \kappa \kappa}^{\dagger}+$ H.c., where the two decoupled sectors labeled by $\kappa$ are related by time-reversal symmetry. Focusing on the first sector (corresponding to $\kappa=1$ ), we find two gapless counterpropagating Majorana modes per DNW that can be written as

$$
\begin{aligned}
& \chi_{L n 1}=\left(e^{-i \pi / 4} \bar{L}_{n 11}+e^{i \pi / 4} \bar{L}_{n 11}^{\dagger}\right) / \sqrt{2}, \\
& \chi_{R n 1}=\left(e^{-i \pi / 4} \bar{R}_{n 1 \overline{1}}+e^{i \pi / 4} \bar{R}_{n 1 \overline{1}}^{\dagger}\right) / \sqrt{2} .
\end{aligned}
$$

Next, we add small interwire hopping terms [see Eq. (4)], where we set $t_{z}=0$ for simplicity. Focusing on the low-energy sector spanned by the states given in Eq. (6), $H_{\perp}$ takes a form similar to a Kitaev chain [68] of coupled 1D modes,

$$
\begin{aligned}
H_{\perp}= & \frac{i}{2} \sum_{n=1}^{N-1} \int d x\left[\left(\beta-\Delta_{c}\right) \chi_{R(n+1) 1} \chi_{L n 1}\right. \\
& \left.+\left(\beta+\Delta_{c}\right) \chi_{L(n+1) 1} \chi_{R n 1}\right],
\end{aligned}
$$

where $N$ is the number of DNWs. At the special point $\Delta_{c}=\beta$, the modes $\chi_{L 11}$ and $\chi_{R N 1}$ do not enter $H_{\perp}$ and thus stay gapless in contrast to all other bulk modes. Obviously, the same is true for their time-reversal partners $\chi_{R 1 \overline{1}}$ and $\chi_{L N \overline{1}}$. Thus, the system is in a helical topological superconducting phase with Kramers partners of gapless Majorana modes propagating along the edges. Even though this result was derived using a considerable amount of fine tuning, the topological properties of the system remain qualitatively identical for a broad range of parameters as long as the bulk gap does not close. In particular, our results do not change if a small $t_{z}$ is included [see Fig. 2(a)].

If, on the other hand, the system is infinite along the $z$ axis and finite along the $x$ axis, we apply the standard procedure of matching decaying eigenfunctions [69] to find a Kramers pair of gapless Majorana edge modes propagating perpendicular to the DNWs [see the Supplemental Material (SM) [70] for details and again Fig. 2(a) for a numerical confirmation].

Fractional helical topological superconducting phase. Now we focus on the fractional counterpart of the helical superconducting phase discussed above. We tune the chemical potential to a fractional value $\mu / E_{s o}=-1+1 / \mathrm{m}^{2}$, where $m$ is an odd integer. The new Fermi momenta are now given by

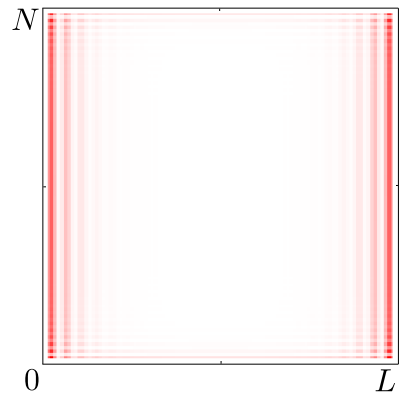

(a)

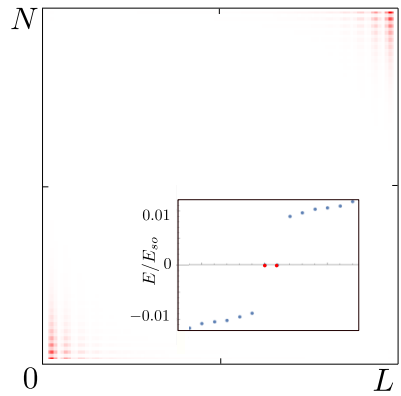

(b)
FIG. 2. Probability density of low-energy states of $H$ [see Eqs. (1)-(4)] obtained numerically. (a) For $\Delta_{Z}=0$, the system is a helical TSC with Kramers partners of gapless Majorana modes propagating along the edges. (b) In the presence of a small in-plane magnetic field, $\Delta_{Z}>0$, we find Majorana bound states localized at two opposite corners of the system. The inset shows the spectrum confirming that these two states (red dots) are indeed at zero energy. The numerical parameters are $N=100, \mu=0, k_{s o} L=85$, $\Gamma / E_{s o} \approx 0.6, \Delta / E_{s o} \approx 0.55, t_{z} / E_{s o} \approx 0.01, \beta / E_{s o} \approx 0.28, \Delta_{c} / E_{s o} \approx$ 0.11 , and, in (b), $\Delta_{Z} / E_{s o} \approx 0.07$ and $\phi=-\pi / 16$. We note that the found topological phases are stable against disorder and do not rely on spatial symmetries.

$k_{F}^{r \tau \sigma}=(\tau \sigma+r / m) k_{s o}$. For $m>1$, the interlayer tunneling term given in Eq. (3) no longer conserves momentum and is therefore suppressed. However, momentum-conserving terms can be constructed by including backscattering terms arising from electron-electron interactions $[2,71]$. These terms are given by $\tilde{H}_{\Gamma, n}=i \tilde{\Gamma} \sum_{\kappa} \int d x\left(\bar{R}_{n \kappa \bar{\kappa}}^{\dagger} \bar{L}_{n \kappa \bar{\kappa}}\right)^{k}$ $\left(\bar{R}_{n \kappa \bar{\kappa}}^{\dagger} \bar{L}_{n \kappa \kappa}\right)\left(\bar{R}_{n \kappa \kappa}^{\dagger} \bar{L}_{n \kappa \kappa}\right)^{k}+$ H.c., $\quad$ where $\quad k=(m-1) / 2$. Similarly, we can write down a dressed superconducting term $\tilde{H}_{\Delta, n}=\tilde{\Delta} \sum_{\kappa, \nu} \kappa v \int d x\left(\bar{R}_{n \kappa \bar{\nu}}^{\dagger} \bar{L}_{n \kappa \bar{\nu}}\right)^{k}\left(\bar{R}_{n \kappa \bar{\nu}}^{\dagger} \bar{L}_{n \kappa \nu}^{\dagger}\right)\left(\bar{R}_{n \kappa \nu} \bar{L}_{n \kappa \nu}^{\dagger}\right)^{k}+$ H.c. Here, the coupling constants $\tilde{\Gamma} \propto \Gamma g_{B}^{m-1}$ and $\tilde{\Delta} \propto$ $\Delta g_{B}^{m-1}$, where $g_{B}$ is the strength of a single backscattering process caused by electron-electron interactions, are assumed to be large $[15,50,51,72]$. In order to treat the interacting Hamiltonian analytically, we adapt a bosonized language $\quad[71]: \quad \bar{R}_{n \kappa v}(x)=e^{i \phi_{1 n \kappa v}(x)}, \quad \bar{L}_{n \kappa v}(x)=e^{i \phi_{\overline{I n k v}}(x)}$ for bosonic fields $\phi_{r n \kappa v}(x)$ satisfying standard nonlocal commutation relations. The dressed superconducting and tunneling terms can be simplified by introducing new bosonic operators $\eta_{r n \kappa v}(x)=\frac{m+1}{2} \phi_{r n \kappa v}(x)-\frac{m-1}{2} \phi_{\bar{r} n \kappa v}(x)$ obeying the commutation relations $\left[\eta_{r n \kappa v}(x), \eta_{r^{\prime} n^{\prime} \kappa^{\prime} v^{\prime}}\left(x^{\prime}\right)\right]=$ $i \pi r m \delta_{r r^{\prime}} \delta_{n n^{\prime}} \delta_{\kappa \kappa^{\prime}} \delta_{v v^{\prime}} \operatorname{sgn}\left(x-x^{\prime}\right)$. The DNW Hamiltonian takes the simple form

$$
\begin{aligned}
H_{n}= & H_{0, n}+2 \sum_{\kappa} \int d x\left[\tilde{\Gamma} \sin \left(\eta_{1 n \kappa \bar{\kappa}}-\eta_{\overline{1} n \kappa \kappa}\right)\right. \\
& \left.+\tilde{\Delta} \cos \left(\eta_{1 n \kappa \bar{\kappa}}+\eta_{\overline{1} n \kappa \kappa}\right)+\tilde{\Delta} \cos \left(\eta_{1 n \kappa \kappa}+\eta_{\overline{1} n \kappa \bar{\kappa}}\right)\right]
\end{aligned}
$$

with $H_{0, n}=\frac{v}{4 \pi m} \sum_{r, \kappa, v} \int d x\left(\partial_{x} \eta_{r n \kappa v}\right)^{2}$, where $v$ is the Fermi velocity and we focus on the special values of Luttinger liquid (LL) parameters $K_{n \kappa \nu}=1 / \mathrm{m}$. Again, half the modes are fully gapped by superconductivity, while for the other modes superconductivity and interlayer tunneling compete. 
Introducing canonically conjugate fields $\varphi_{n \kappa}=\left(\eta_{1 n \kappa \bar{\kappa}}-\right.$ $\left.\eta_{\overline{1} n \kappa \kappa}-\pi / 2\right) /(2 \sqrt{m}), \quad \theta_{n \kappa}=\left(\eta_{1 n \kappa \bar{\kappa}}+\eta_{\overline{1} n \kappa \kappa}\right) /(2 \sqrt{m})$, the competing part of the above Hamiltonian can be rewritten as

$$
\begin{aligned}
H_{n}= & \sum_{\kappa} \int d x\left\{\frac{v}{2 \pi}\left[\left(\partial_{x} \varphi_{n \kappa}\right)^{2}+\left(\partial_{x} \theta_{n \kappa}\right)^{2}\right]\right. \\
& \left.+2 \tilde{\Gamma} \cos \left(2 \sqrt{m} \varphi_{n \kappa}\right)+2 \tilde{\Delta} \cos \left(2 \sqrt{m} \theta_{n \kappa}\right)\right\} .
\end{aligned}
$$

For $\tilde{\Gamma}=\tilde{\Delta}$, this Hamiltonian corresponds to two timereversed copies of a well-known self-dual sine-Gordon model $[73,74]$. For $m=1$, we thus expect to find a single gapless Majorana mode per time-reversal sector, which is consistent with our analysis of the noninteracting regime in the previous section. To study the more general case, we start by noting that for our choice of LL parameters, the competing terms have the same scaling dimension, which allows us to explicitly study the properties of the system along the self-dual line. For $m>1$, however, the superconducting and tunneling terms are irrelevant to first order in the renormalization group (RG) analysis, suggesting a flow to a trivial LL fixed point. To resolve this issue, Ref. [72] argued that upon including a thirdorder term in the RG equations, a multicritical fixed point is encountered, which in our case separates a gapless phase, a phase dominated by superconductivity, and a phase dominated by interlayer tunneling. Such a fixed point has been shown to be described by a $\mathbb{Z}_{2 m}$ parafermion theory [74], which means in our case that there are two bulk $\mathbb{Z}_{2 m}$ parafermion modes related by time-reversal symmetry residing within each DNW.

We now refermionize the above model in order to obtain an explicit expression for specific primary fields [74] of these parafermion theories. In particular, we define new composite chiral fermion operators $\bar{\psi}_{n \kappa \nu}^{(m)}(x)=\bar{R}_{n \kappa \nu}^{(m)}(x) e^{i q_{F}^{1 \kappa \nu} x}+$ $\bar{L}_{n \kappa \nu}^{(m)}(x) e^{i q_{F}^{\overline{1} \kappa \nu} x}$ with $\bar{R}_{n \kappa \nu}^{(m)}=e^{i \eta_{1 n \kappa \nu}}, \bar{L}_{n \kappa \nu}^{(m)}=e^{i \eta_{\overline{1} n \kappa \nu}}$ and Fermi momenta $q_{F}^{r \kappa \nu}=\frac{m+1}{2} k_{F}^{r \kappa \nu}-\frac{m-1}{2} k_{F}^{\bar{r} \kappa \nu}$ [5]. The superconducting and tunneling terms acting on the interior branches around $q_{F}=0$ then take the forms $H_{\Gamma, n}=i \tilde{\Gamma} \sum_{\kappa} \int d x \bar{R}_{n \kappa \bar{\kappa}}^{(m) \dagger} \bar{L}_{n \kappa \kappa}^{(m)}+$ H.c., $H_{\Delta, n}=\tilde{\Delta} \sum_{\kappa} \int d x \bar{R}_{n \kappa \bar{\kappa}}^{(m) \dagger} \bar{L}_{n \kappa \kappa}^{(m) \dagger}+$ H.c., from which we recover the noninteracting case by setting $m=1$. Forgetting about the underlying model and thinking in terms of the new fermions only, one can perform the same steps as in the noninteracting case to show that the modes

$$
\begin{aligned}
& \chi_{L n 1}^{(m)}=\left(e^{-i \pi / 4} \bar{L}_{n 11}^{(m)}+e^{i \pi / 4} \bar{L}_{n 11}^{(m) \dagger}\right) / \sqrt{2}, \\
& \chi_{R n 1}^{(m)}=\left(e^{-i \pi / 4} \bar{R}_{n 1 \overline{1}}^{(m)}+e^{i \pi / 4} \bar{R}_{n 1 \overline{1}}^{(m) \dagger}\right) / \sqrt{2}
\end{aligned}
$$

commute with the superconducting and tunneling term, and the same is true for their Kramers partners $\chi_{L n \overline{1}}^{(m)}, \chi_{R n \overline{1}}^{(m)}$. The above solutions satisfy $\chi_{r n \kappa}^{(m) \dagger}=\chi_{r n \kappa}^{(m)}$, which prompts us to identify them as the $\psi_{m}$ primary fields of the $\mathbb{Z}_{2 m}$ parafermion theories describing each DNW. Note that these fields are local in terms of electrons, which makes them particularly convenient to handle.

Similar to the noninteracting case, we introduce dressed interwire couplings for $m>1$, which now couple the $\bar{R}_{n \kappa \nu}^{(m)}, \bar{L}_{n \kappa \nu}^{(m)}$ fields. Assuming that the interwire terms are relevant [70] and repeating the analysis of the integer case for the modes given in Eq. (10), we find that the bulk of the system is fully gapped, while there is a Kramers pair of gapless modes propagating along the edges of a finite sample. These modes correspond to $\psi_{m}$ primary fields of a $\mathbb{Z}_{2 m}$ parafermion theory. However, it is expected [42] that there are indeed two full $\mathbb{Z}_{2 m}$ parafermion theories residing at the edges of the system.

Majorana and parafermion corner states. We now show that in the presence of a weak in-plane magnetic field, the system enters a second-order topological superconducting phase. Let us start from the (noninteracting) Zeeman Hamiltonian $H_{Z}=\Delta_{Z} \sum_{n, \tau, \sigma, \sigma^{\prime}} \int d x \psi_{n \tau \sigma}^{\dagger}\left[\cos (\phi)\left(\sigma_{x}\right)_{\sigma \sigma^{\prime}}+\right.$ $\left.\sin (\phi)\left(\sigma_{z}\right)_{\sigma \sigma^{\prime}}\right] \psi_{n \tau \sigma^{\prime}}$. For $m>1$, momentum-conserving terms are once again constructed by including suitable backscattering processes, such that the dressed term then couples the $\bar{R}_{n \kappa \nu}^{(m)}, \bar{L}_{n \kappa \nu}^{(m)}$ fields. In the following, we focus on the regime where the magnetic field strength $\tilde{\Delta}_{Z}$ is small enough not to modify the bulk structure. However, as time-reversal symmetry is broken, the helical edge modes are gapped out. Assuming that the system size is large such that far away from the corners, all four edges can be treated independently, we calculate the projection of $H_{Z}$ onto the edge states for all four edges (see the SM [70]). If we label the edges of a rectangular sample by an index $p=0, \ldots, 3$ in counterclockwise order starting from the bottom edge, the projection of the Zeeman Hamiltonian onto the edge $p$ is given by

$$
\mathcal{H}_{Z}^{\text {eff, } p}=-\tilde{\Delta}_{Z} \cos \left(\phi+\varphi_{p}\right) \gamma_{y},
$$

where we have defined $\varphi_{p}=p \pi / 2$ and $\gamma_{y}$ is a Pauli matrix acting on the low-energy subspace spanned, in this order, by the low-energy edge mode belonging to the time-reversal sector $\kappa=1$ and its Kramers partner belonging to the sector $\kappa=\overline{1}$. This shows that the mass term changes sign at two corners of the system. Explicitly, the sign change occurs at two diagonally opposite corners of the sample depending on the direction of the magnetic field. For $\phi \in(0, \pi / 2) \cup(\pi, 3 \pi / 2)$ $[\phi \in(\pi / 2, \pi) \cup(3 \pi / 2,2 \pi)]$ the sign change occurs at the top-left and bottom-right (top-right and bottom-left) corners. In the spirit of a Jackiw-Rebbi model [75], there are bound states at the corners where the mass term changes sign, which in our case inherit the exotic properties of the propagating modes and thus can be identified as zero-energy $\mathbb{Z}_{2 m}$ parafermion corner states. Again, while this result was derived for the local $\psi_{m}$ fields, we expect that our arguments generalize to the full set of $\mathbb{Z}_{2 m}$ primary fields. In the noninteracting limit $m=1$, we find zero-energy Majorana corner states, which is verified numerically in Fig. 2(b).

Conclusions. We have studied a system consisting of two layers of coupled Rashba nanowires in the presence of interlayer tunneling and proximity-induced superconductivity of a phase difference of $\pi$ between the layers. We have shown that strong electron-electron interactions can stabilize a helical topological superconducting phase exhibiting Kramers partners of gapless $\mathbb{Z}_{2 m}$ parafermion edge modes. Upon turning on a small in-plane magnetic field, the system enters a second-order topological superconducting phase hosting exotic zero-energy parafermion bound states at two corners of a rectangular sample depending on the direction of the magnetic field.

Our analytical approach is limited to the perturbative regime. However, if the system parameters are increased resulting in nonperturbative gaps, the parafermions will still 
be present as long as the bulk gap is not closed. Such nonperturbative regimes could be accessed numerically. Thus, our model provides a proof of principle for the existence of helical fractional TSCs and second-order fractional TSCs and we envision it to be a representative of a more general class of systems exhibiting the same parafermionic features.
Acknowledgments. This work was supported by the Swiss National Science Foundation and NCCR QSIT. This project received funding from the European Unions Horizon 2020 research and innovation program (ERC Starting Grant, Grant Agreement No 757725). We acknowledge helpful discussions with Yanick Volpez.
[1] C. L. Kane, R. Mukhopadhyay, and T. C. Lubensky, Phys. Rev. Lett. 88, 036401 (2002).

[2] J. C. Y. Teo and C. L. Kane, Phys. Rev. B 89, 085101 (2014).

[3] J. Klinovaja and D. Loss, Eur. Phys. J. B 87, 171 (2014).

[4] J. Klinovaja and Y. Tserkovnyak, Phys. Rev. B 90, 115426 (2014).

[5] E. Sagi and Y. Oreg, Phys. Rev. B 90, 201102(R) (2014).

[6] T. Meng and E. Sela, Phys. Rev. B 90, 235425 (2014).

[7] T. Neupert, C. Chamon, C. Mudry, and R. Thomale, Phys. Rev. B 90, 205101 (2014).

[8] J. Klinovaja, Y. Tserkovnyak, and D. Loss, Phys. Rev. B 91, 085426 (2015).

[9] T. Meng, Phys. Rev. B 92, 115152 (2015).

[10] E. Sagi and Y. Oreg, Phys. Rev. B 92, 195137 (2015).

[11] R. A. Santos, C.-W. Huang, Y. Gefen, and D. B. Gutman, Phys. Rev. B 91, 205141 (2015).

[12] J. Klinovaja, P. Stano, and D. Loss, Phys. Rev. Lett. 116, 176401 (2016).

[13] S. Sahoo, Z. Zhang, and J. C. Y. Teo, Phys. Rev. B 94, 165142 (2016).

[14] A. Vaezi, Phys. Rev. X 4, 031009 (2014).

[15] E. Sagi, A. Haim, E. Berg, F. von Oppen, and Y. Oreg, Phys. Rev. B 96, 235144 (2017).

[16] T. Meng, T. Neupert, M. Greiter, and R. Thomale, Phys. Rev. B 91, 241106(R) (2015).

[17] G. Gorohovsky, R. G. Pereira, and E. Sela, Phys. Rev. B 91, 245139 (2015).

[18] P.-H. Huang, J.-H. Chen, P. R. S. Gomes, T. Neupert, C. Chamon, and C. Mudry, Phys. Rev. B 93, 205123 (2016).

[19] W. A. Benalcazar, J. C. Y. Teo, and T. L. Hughes, Phys. Rev. B 89, 224503 (2014).

[20] W. A. Benalcazar, B. A. Bernevig, and T. L. Hughes, Science 357, 61 (2017).

[21] W. A. Benalcazar, B. A. Bernevig, and T. L. Hughes, Phys. Rev. B 96, 245115 (2017).

[22] Z. Song, Z. Fang, and C. Fang, Phys. Rev. Lett. 119, 246402 (2017).

[23] Y. Peng, Y. Bao, and F. von Oppen, Phys. Rev. B 95, 235143 (2017).

[24] S. Imhof, C. Berger, F. Bayer, H. Brehm, L. Molenkamp, T. Kiessling, F. Schindler, C. H. Lee, M. Greiter, T. Neupert, and R. Thomale, Nat. Phys. 14, 925 (2018).

[25] M. Geier, L. Trifunovic, M. Hoskam, and P. W. Brouwer, Phys. Rev. B 97, 205135 (2018).

[26] F. Schindler, A. M. Cook, M. G. Verginory, Z. Wang, S. S. P. Parking, B. A. Bernevig, and T. Neupert, Sci. Adv. 4, 6 (2018).

[27] C.-H. Hsu, P. Stano, J. Klinovaja, and D. Loss, Phys. Rev. Lett. 121, 196801 (2018).

[28] M. Ezawa, Sci. Rep. 9, 5286 (2019).

[29] M. Ezawa, Phys. Rev. Lett. 121, 116801 (2018).
[30] X. Zhu, Phys. Rev. B 97, 205134 (2018).

[31] Q. Wang, C.-C. Liu, Y.-M. Lu, and F. Zhang, Phys. Rev. Lett. 121, 186801 (2018).

[32] Z. Yan, F. Song, and Z. Wang, Phys. Rev. Lett. 121, 096803 (2018).

[33] T. Liu, J. J. He, and F. Nori, Phys. Rev. B 98, 245413 (2018).

[34] X. Zhang, H.-X. Wang, Z.-K. Lin, Y. Tian, B. Xie, M.-H. Lu, Y.-F. Chen, and J.-H. Jiang, Nat. Phys. 15, 582 (2019).

[35] Q. Wang, D. Wang, and Q.-H. Wang, Europhys. Lett. 124, 50005 (2018).

[36] Y. Volpez, D. Loss, and J. Klinovaja, Phys. Rev. Lett. 122, 126402 (2019).

[37] A. Agarwala, V. Juricic, and B. Roy, arXiv:1902.00507.

[38] D. Calugaru, V. Juricic, and B. Roy, Phys. Rev. B 99, 041301(R) (2019).

[39] M. Kheirkhah, Y. Nagai, C. Chen, and F. Marsiglio, arXiv:1904.00990.

[40] Y. You, D. Litinski, and F. von Oppen, Phys. Rev. B 100, 054513 (2019).

[41] Y. You, T. Devakul, F. J. Burnell, and T. Neupert, Phys. Rev. B 98, 235102 (2018)

[42] R. S. K. Mong, D. J. Clarke, J. Alicea, N. H. Lindner, P. Fendley, C. Nayak, Y. Oreg, A. Stern, E. Berg, K. Shtengel, and M. P. A. Fisher, Phys. Rev. X 4, 011036 (2014).

[43] N. H. Lindner, E. Berg, G. Refael, and A. Stern, Phys. Rev. X 2, 041002 (2012).

[44] D. J. Clarke, J. Alicea, and K. Shtengel, Nat. Commun. 4, 1348 (2013).

[45] M. Cheng, Phys. Rev. B 86, 195126 (2012).

[46] J. Klinovaja, A. Yacoby, and D. Loss, Phys. Rev. B 90, 155447 (2014).

[47] C. P. Orth, R. P. Tiwari, T. Meng, and T. L. Schmidt, Phys. Rev. B 91, 081406(R) (2015).

[48] J. Klinovaja and D. Loss, Phys. Rev. B 92, 121410(R) (2015).

[49] C. Fleckenstein, N. T. Ziani, and B. Trauzettel, Phys. Rev. Lett. 122, 066801 (2019).

[50] Y. Oreg, E. Sela, and A. Stern, Phys. Rev. B 89, 115402 (2014).

[51] J. Klinovaja and D. Loss, Phys. Rev. Lett. 112, 246403 (2014).

[52] J. Klinovaja and D. Loss, Phys. Rev. B 90, 045118 (2014).

[53] C. J. Pedder, T. Meng, R. P. Tiwari, and T. L. Schmidt, arXiv:1507.08881.

[54] M. Thakurathi, D. Loss, and J. Klinovaja, Phys. Rev. B 95, 155407 (2017).

[55] F. Iemini, C. Mora, and L. Mazza, Phys. Rev. Lett. 118, 170402 (2017).

[56] A. Calzona, T. Meng, M. Sassetti, and T. L. Schmidt, Phys. Rev. B 98, 201110(R) (2018).

[57] L. Mazza, F. Iemini, M. Dalmonte, and C. Mora, Phys. Rev. B 98, 201109(R) (2018). 
[58] H. Ren, F. Pientka, S. Hart, A. T. Pierce, M. Kosowsky, L. Lunczer, R. Schlereth, B. Scharf, E. M. Hankiewicz, L. W. Molenkamp, B. I. Halperin, and A. Yacoby, Nature (London) 569, 93 (2019).

[59] A. Fornieri, A. M. Whiticar, F. Setiawan, E. Portolés, A. C. C. Drachmann, A. Keselman, S. Gronin, C. Thomas, T. Wang, R. Kallaher, G. C. Gardner, E. Berg, M. J. Manfra, A. Stern, C. M. Marcus, and F. Nichele, Nature (London) 569, 89 (2019).

[60] C. Schrade, A. A. Zyuzin, J. Klinovaja, and D. Loss, Phys. Rev. Lett. 115, 237001 (2015).

[61] A. I. Buzdin, L. N. Bulaevskii, and S. V. Panyukov, Pis'ma Zh. Eksp. Teor. Fiz. 35, 147 (1982) [JETP Lett. 35, 178 (1982)].

[62] V. V. Ryazanov, V. A. Oboznov, A. Yu. Rusanov, A. V. Veretennikov, A. A. Golubov, and J. Aarts, Phys. Rev. Lett. 86, 2427 (2001).

[63] B. I. Spivak and S. A. Kivelson, Phys. Rev. B 43, 3740 (1991).

[64] J. A. van Dam, Y. V. Nazarov, E. P. A. M. Bakkers, and L. P. Kouwenhoven, Nature (London) 442, 667 (2006).

[65] S. Ryu, A. P. Schnyder, A. Furusaki, and A. W. W. Ludwig, New J. Phys. 12, 065010 (2010).

[66] J. Klinovaja and D. Loss, Phys. Rev. B 86, 085408 (2012).
[67] The assumption of equal Fermi momenta for all nanowires can be relaxed to the requirement that the Fermi momenta of only the interior branches are approximately the same. While small deviations from this point do not alter our main conclusions, substantial differences suppress the interlayer tunneling due to momentum mismatch.

[68] A. Y. Kitaev, Phys. Usp. 44, 131 (2001).

[69] J. Klinovaja and D. Loss, Phys. Rev. Lett. 111, 196401 (2013).

[70] See Supplemental Material at http://link.aps.org/supplemental/ 10.1103/PhysRevResearch.1.032017 for details regarding the interwire couplings in the interacting case, an explicit calculation of the edge state wave functions perpendicular to the wires, as well as the derivation of the effective low-energy edge Hamiltonian.

[71] T. Giamarchi, Quantum Physics in One Dimension (Oxford University Press, Oxford, U.K., 2004).

[72] D. Boyanovsky, J. Phys. A: Math. Gen. 22, 2601 (1989).

[73] P. Lecheminant, A. O. Gogolin, and A. A. Nersesyan, Nucl. Phys. B 639, 502 (2002).

[74] A. B. Zamolodchikov and V. A. Fateev, Zh. Eksp. Teor. Fiz. 89, 380 (1985).

[75] R. Jackiw and C. Rebbi, Phys. Rev. D 13, 3398 (1976). 